\title{
IMPLEMENTACIÓN DE PROCEDIMIENTOS DE GOBERNABILIDAD TI EN LA RED DE INVESTIGACIÓN DE TECNOLOGÍA AVANZADA BASADO EN ITIL, COBIT Y LA ISO 20000-27000
}

\section{IMPLEMENTATION OF IT GOVERNANCE PROCEDURES RESEARCH NETWORK FOR ADVANCED TECHNOLOGY BASED ON ITIL, COBIT AND ISO 20000-27000}

\section{RESUMEN}

La implementación de la gobernabilidad de las Tecnologías de la Información TI es esencial en todo tipo de organización, por lo tanto es necesaria su aplicación en la Red de Investigación de Tecnología Avanzada de la Universidad Distrital Francisco José de Caldas para lograr la alineación de las TI con los objetivos globales de esta, como apoyo en todas sus áreas. Para ello se debe efectuar la práctica de modelos organizacionales como ITIL, COBIT, ISO 20000 e ISO 27000.

Palabras clave: Gobernabilidad, ITL, COBIT, ISO20.000-27000.

\section{ABSTRACT}

The implementation of IT Governance (Information Technology) is essential in any type of organization, it is therefore necessary application to the High Speed Research Network in the District University Francisco José de Caldas to achieve alignment of IT with the overall objectives of this, as support in all areas. This requires making practice organizational models like ITIL, COBIT, ISO 20000 and ISO 27000

Keywords: Governability, ITL, COBIT, ISO20.000-27000.

\section{INTRODUCCIÓN}

Actualmente para muchas organizaciones, entre ellas las universidades como centros de formación, investigación e innovación de nuevos esquemas organizacionales y de gobernabilidad basados en TI, la información y la tecnología que las soportan representan sus activos intangibles más significativos, activos que con frecuencia son poco entendidos por su comunidad. Estas instituciones consideran los beneficios que aportan la tecnología de información por lo cual es importante su administración a nivel gerencial.

El gobierno de TI en las universidades debe centrarse en conseguir una gestión eficiente de los recursos tecnológicos como soporte principal de los servicios universitarios. De esta manera, una buena im-

\section{Roberto Ferro Escobar: \\ Ingeniero Electrónico - Universidad Distrital Francisco José de Caldas - Colombia. Doctor en gestión del co- nocimiento - UPSAM - España. \\ Decano facultad de Ingeniería - Uni- versidad Distrital Francisco José de Caldas - Colombia - rferro@udistri- tal.edu.co, ferro.roberto@gmail.com}

Giovanny Mauricio Tarazona Bermúdez:

Ingeniero Industrial - Universidad Distrital Francisco José de Caldas Colombia. Doctor para sistemas informáticos para internet - Universidad de Oviedo - España.

Docente - Universidad Distrital Francisco José de Caldas - Colombia Gtarazona@udistrital.edu.co, Gtarazona@gmail.com

\section{Gabriel Andres Alzate Acuña:}

Ingeniero Electrónico - Universidad Distrital Francisco José de Caldas - Colombia. gaalzatea@correo.udistrital.edu.co, gabriel.andres.alzate@ gmail.com

Tipo: Reporte de caso

Fecha de Recepción: Abril 30 de 2015

Fecha de Aceptación: Junio 24 de 2015 
plementación de procedimientos del gobierno de las TI basados en estándares posibilita que las universidades lleven a cabo una proyección estratégica de las tecnologías de la información de manera alineada con los objetivos globales de la organización.

De aquí, que la planificación de las TI debe ser responsabilidad directa de la alta dirección universitaria. Adicional a esto la directiva debe mejorar el uso de los recursos disponibles, como aplicaciones, información, infraestructura y personas. En este orden de ideas, la universidad debe adoptar procedimientos de gobernabilidad basados en estos importantes aspectos. El objetivo de este artículo es sentar las pautas adecuadas basadas en los estándares de ITIL, COBIT y la ISO para implementar un modelo en la Red de Investigación de Tecnología Avanzada de la Universidad Distrital Francisco José de Caldas procesos de gobernabilidad TI.

Contexto De La Universidad Distrital Francisco José De Caldas

La Universidad Distrital Francisco José de Caldas por ser una institución pública de educación superior, cuya misión propone: "Es la calificación de egresados con capacidad de actuar como protagonistas del cambio social y de sí mismo, en la formación del espíritu científico aplicado a la indagación, interpretación y modificación de la realidad y en la contribución a forjar ciudadanos idóneos para promover el progreso de la sociedad." [1]

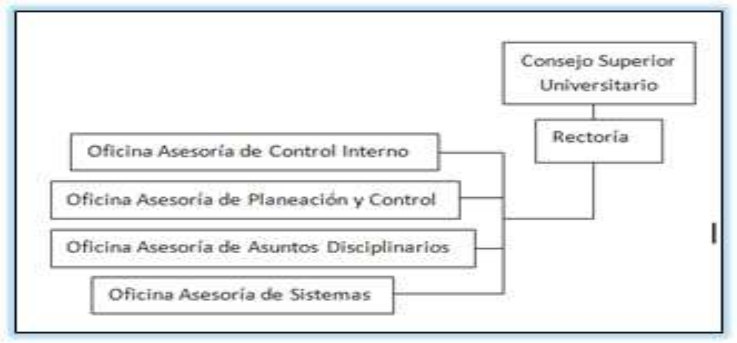

Figura. 1 Organigrama de la UDFJC de la OAS [1]

En la Figura 1 se observa parte del organigrama de gobernabilidad la Universidad, de allí se puede observar que la oficina asesoría de sistemas reporta directamente a la rectoría y está alineada con las oficinas de control interno, planeación y control y asuntos disciplinarios.

Recursos de TI: Actualmente la Universidad existen cuatro tipos de sistemas de información, los transaccionales ya por desarrollo propio $o$ adquirido por terceros, que permiten organizar los datos y la información para apoyar los procesos misionales de la institución estos son CÓNDOR, SICAPITAL, SICIUD, ILUD y ALE$\mathrm{PH}$, los de tipo gerencial o estratégico que permiten realizar análisis y estadísticas a la información transaccional con el fin de ser insumo para la toma de decisiones (Bodega de Datos, ICARO y SIAUD), los sistemas de tipo netamente académicos especializados y que son fruto de la Docencia, la Investigación y la Extensión y el sistema que integra los sistemas transaccionales en un sistema único.

Dentro de la normatividad y los parámetros de desarrollo de la Universidad Distrital se ha creado este Plan Estratégico que como elemento directriz en los procesos de planeación y mejoramiento, dentro de la autonomía y el manejo eficiente de recurso tal como se señala en el mismo documento; en el capítulo relacionada con Políticas, Estrategias, Programas, Proyectos y Metas, se destaca la Política 6: "Desarrollo físico e infraestructura tecnológica de la Universidad", donde se formula el siguiente objetivo específico: "Mejorar y mantener actualizada la infraestructura física y tecnológica de la Universidad en función de la proyección de las actividades misionales de la Universidad." Para dar cumplimiento a este propósito se plantea las siguientes pautas:

Estrategia 1. Desarrollo y actualización permanente de la infraestructura física, tecnológica, de conectividad y de recursos en general. Consolidar, fortalecer y mantener actualizada la infraestructura física y de servicios informáticos y de comunicaciones así como la adecuación, construcción y dotación de espacios para el ejercicio de actividades en relación con las funciones universitarias.

-Proyecto 1: Crear y definir la arquitectura del 
Sistema de Información y comunicación interno y externo.

-Proyecto 2: Fortalecer, adecuar y dotar la infraestructura de comunicaciones e información y conectividad.

-Proyecto 3: Masificar el uso de tecnologías de comunicación e información.

Debido a lo anterior y en concordancia con lo formulado en la política 6 en el año 2008 el CSU para mejorar los temas relacionados con la conectividad a alta velocidad y la articulación con Redes académicas tipo Internet2 [2] como RUMBO [3], RENATA [4] y CLARA [5] creo la Red de Investigación de Tecnología Avanzada "RITA"[6]; de allí se fortalece el uso de las TIC, la gobernabilidad basada en estándares internacionales y el cumplimiento del objeto misional de la universidad basado en sus tres pilares, la academia, la docencia y la investigación.

\section{Gobierno De Las TI}

Existen variadas definiciones del gobierno TI. Según el IT Governance Institute dice: "El gobierno de las TI es responsabilidad de la administración ejecutiva y del Consejo de Dirección. Es una parte integral del gobierno de una organización y consiste en las estructuras organizacionales y de dirección, y en los procesos que aseguran que la organización mantiene y amplía sus objetivos y estrategias" [7]. Se puede decir entonces que su principal objetivo es conseguir la alineación entre la estrategia del negocio y las TI y de esta manera minimizar los riesgos.

En la siguiente Figura se encuentran las cinco áreas del enfoque del gobierno TI.

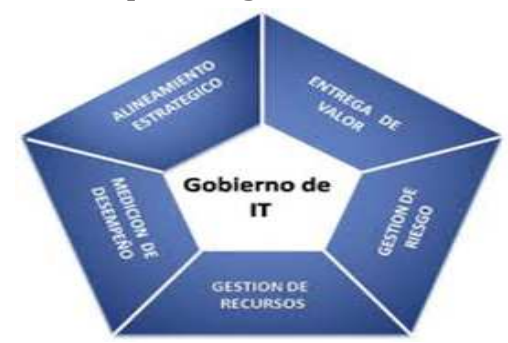

Figura 2. Áreas de Enfoque del Gobierno de TI
Podemos decir que el gobierno de las TI se puede ser aplicado a la Red RITA como elemento para crear valor al conjunto de la comunidad universitaria y la sociedad en la que se enmarca su actuación.

\section{MODELOS DE GOBERNABILIDAD DE TI}

Actualmente existen diferentes modelos orientados al control de las organizaciones, cada una de ellas abarca diferentes campos de acción, de forma que se complementan. Están relacionados con la toma de decisiones estratégicas, la planificación estratégica de los sistemas de información, la gestión de los servicios, y con las herramientas de monitorización y control. A continuación veremos: ITIL [9], COBIT [10], ISO 20000 [11] y ISO 27000 [12], y su implementación en la Universidad Distrital [13].

A. ITIL (Information Technology and Infraestructure Library)

Es una guía de las prácticas de cómo diseñar, desarrollar e implementar una Gestión de Servicios como un activo estratégico, basado en procesos. La Figura 2 muestra los procesos en el ciclo de vida de ITIL.

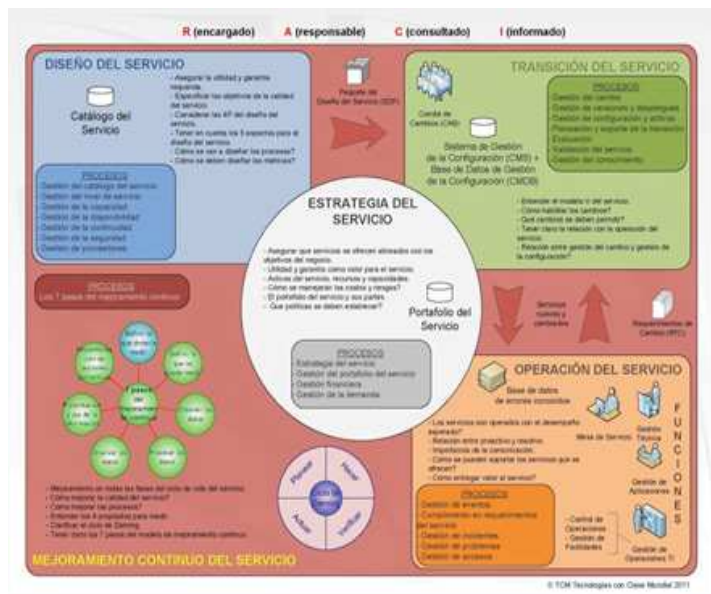

Figura 3. Ciclo de Vida ITIL y sus funciones primordiales. [14]

Según las recomendaciones de ITIL se realizara a continuación la implementación para la Universidad Distrital. Se realizó algunos subprocesos de la Gestión del Diseño de servicios, Transición del servicio y la Operación del servicio. 


\section{DISEÑO DEL SERVICIOS}

En esta fase se realizaron dos procesos la gestión de catálogo de servicios y la gestión de la seguridad de la información.

Catálogo de Servicios: Primero se debe caracterizar y catalogar los servicios de tal forma que se pueda conocer los siguientes campos en un formato:

La descripción del servicio, acuerdos de Nivel de Servicio, quienes están autorizados para requerir o ver el servicio, el costo del servicio y la forma de cumplir el servicio.

En la tabla 1, se puede ver un ejemplo del formato del servicio RITA de la Universidad Distrital.

Tabla I. Formato de catalogio de servicios RITAUD:

\begin{tabular}{|l|l|l|}
\hline \multicolumn{2}{|c|}{ FORMATO CATALOGO DE SERVCIOS RITA } & FCSO001 \\
\hline Servicio: & Préstamo de servicios de alta conectividad \\
\hline Descripción: & $\begin{array}{l}\text { Servicio que se le presta a la comunidad } \\
\text { académica de la universidad relacionada con la } \\
\text { articulación con Redes de Alta Velocidad. }\end{array}$ \\
\hline Responsable: & Gobiemo RT A-UD \\
\hline Horario: & $\begin{array}{l}\text { De Lunes a Viernes, de 6.00 am a 10.00 pm } \\
\text { Observaciones: }\end{array}$ & $\begin{array}{l}\text { El proceso para solicitar este servicio IT es el } \\
\text { siguiente: } \\
\text { 1. Diligenciar vía web el formato de servicio. } \\
\text { 2. Elaborar los respectivos acuerdos indicando } \\
\text { naturaleza de la actividad, hora y tiempo durante } \\
\text { el cual se van a utilizar, el tipo de software que se } \\
\text { necesita con el visto bueno de la unidad } \\
\text { encargada o coordinador. } \\
\text { 3. Una vez recibida la solicitud en la coordinación } \\
\text { de RITA-UD, se procederá a la oficialización del } \\
\text { requerimiento y su respuesta. }\end{array}$ \\
\hline
\end{tabular}

Seguridad de la Información: En la Universidad Distrital se ha implementado una política para la seguridad de la información donde estipula su responsabilidad con el proceso de gestión responsable de información que tiene como objetivo garantizar la integridad, confidencialidad y disponibilidad de este significativo activo, teniendo como eje el cumplimiento de los objetivos misionales.

Su objetivo primordial "Proteger, preservar y administrar objetivamente la información de la Universidad Distrital Francisco José de Caldas junto con las tecnologías utilizada para su procesamiento, frente a amenazas internas o externas, deliberadas o accidentales, con el fin de asegurar el cumplimiento de los características de confidencialidad, integridad, disponibilidad, legalidad, confiabilidad y no repudio de la información" [15].

\section{TRANSICIÓN DEL SERVICIO}

En esta fase se establecen expectativas del cliente de cómo debe ser prestado el servicio y su uso, además de garantizar que sean accesibles a los usuarios autorizados. Por lo cual se debe manejar el proceso de gestión de cambios. Gestión de cambios: Para llevar un mayor control en los servicios de la Universidad en cuanto a los cambios, se debe solicitar por medio del siguiente formato:

Tabla. II. Formato de solicitus de cambios UD

\begin{tabular}{|c|c|c|}
\hline \multicolumn{2}{|c|}{ FORMATO DE SOUCITUD DE CAMBIS } & FSCOOO1 \\
\hline Fecha: & \multicolumn{2}{|l|}{ Febrero 2014} \\
\hline Responsable: & \multicolumn{2}{|c|}{ Decano Ingenieria } \\
\hline Área: & \multicolumn{2}{|l|}{ Maestría CIC } \\
\hline \multicolumn{3}{|c|}{ Clasificación del cambio según servicio (Marque una X) } \\
\hline Sistemas de Información & \multicolumn{2}{|c|}{ Correo Electrónico: } \\
\hline Servidores & \multicolumn{2}{|c|}{ Otro: Video Conferencia } \\
\hline Prioridad: & \multicolumn{2}{|l|}{ Alta } \\
\hline Descripción del Cambio: & & \\
\hline Motivo del Cambio: & & \\
\hline
\end{tabular}

La gestión de cambios ayuda a los gestores de IT y al personal de IT a mantener informados a la directiva de la Universidad y a las partes in- 
teresadas en los momentos en que tienen lugar cambios importantes.

\section{OPERACIÓN DEL SERVICIO}

Esta etapa es la entrega al usuario con la mejor calidad del servicio TI, por lo cual se debe efectuar una gestión de implementaciones con el objetivo de planificar y realizar los cambios sin contratiempos y de manera transparente para el usuario.

Gestión de Implementaciones: Esta va de la mano con la gestión de cambios, así que debe realizarse un plan de pruebas en un entorno real y luego un plan de implementación con información sobre lo que se va a implantar, cómo se va a implantar y las especificaciones en las que funcionará [15].

Tabla III. Formato de plan de prueba RITA-UD

\begin{tabular}{|c|c|}
\hline FORMATO DE PLAN DE PRUEBAS & FPP0001 \\
\hline Caso de Prueba: & \\
\hline Propósito: & \\
\hline $\begin{array}{l}\text { Prerrequisitos: } \\
\text { Datos de Prueba: }\end{array}$ & \\
\hline Pasos: & \\
\hline Notas y Pregunta 5: & \\
\hline
\end{tabular}

\section{COBIT (CONTROL OBJECTIVES FOR INFORMATION AND RELATED TECHNOLOGY)}

Es un modelo para verificar la gestión y control de los sistemas de información y tecnología, encaminado a todas las áreas de una organización, es decir, administradores TI, usuarios y por supuesto, los auditores implicados en el proceso.

COBIT se basa en el siguiente principio "Para proporcionar la información que la empresa requiere para lograr sus objetivos, la empresa necesita invertir, administrar y controlar los recursos de TI usando un conjunto estructurado de procesos que provean los servicios que entregan la información empresarial requerida." [7].

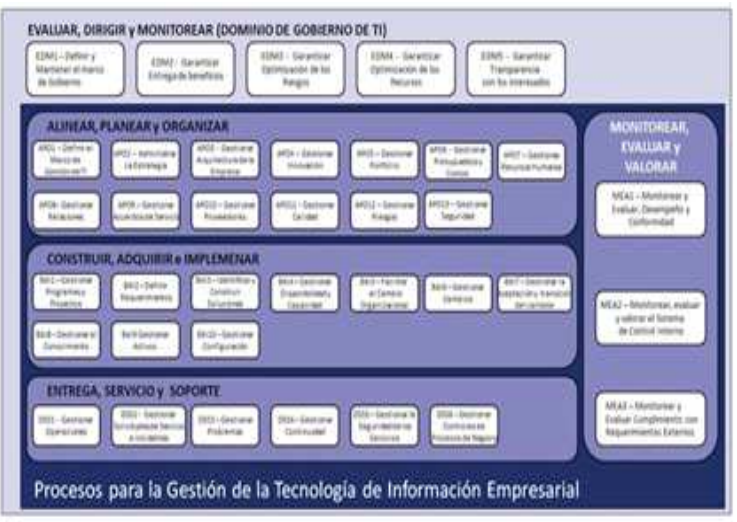

Figura 4. Principio de COBIT y su implementación [7].

Para empezar la implementación de COBIT en RITA-UD, se ha utilizado la matriz de riesgos efectuada por ISACA. Esta matriz nos permite identificar los procesos y actividades más críticos. Se tomó como referencia el Plan Maestro de Informática y Telecomunicaciones [13] para la calificación de los aspectos ya que RITA se encuentra en uno de los sub procesos.

Los procesos que son prioridad según el resultado arrojado por la matriz de la tabla 4:

- $\quad$ P01 Definir un plan estratégico de TI

- $\quad$ P04 Definir los procesos, organización y relaciones de TI

P05 Administrar la inversión en TI

- $\quad$ P09 Evaluar y administrar los riesgos de TI

- $\quad$ AI2 Adquirir y mantener software apli cativo

- $\quad$ AI3 Adquirir y mantener infraestructu ra tecnológica

- $\quad$ AI4 Facilitar la operación y el uso

- $\quad$ DS4 Garantizar la continuidad del ser vicio

- $\quad$ DS5 Garantizar la seguridad de los sis temas 
- $\quad$ DS7 Educar y entrenar a los usuarios

- $\quad$ ME1 Monitorear y evaluar el desempeño de TI

Tabla IV. Matriz de riesgos

\begin{tabular}{|c|c|c|}
\hline PROCESOS DE TI & Importancia: 1 Menos importante, 5 más & Impacto: Alto, Medio, Bajo, Inmaterial, No \\
\hline \multicolumn{3}{|c|}{ PLANEAR Y ORGANIZAR } \\
\hline P01 Definir un plan estratégico de TI & 5 & Alto \\
\hline PO2 Definir la arquitectura de la información & 2 & Bajo \\
\hline PO3 Determinar la dirección tecnológica & 1 & Inmaterial \\
\hline PO4 Definir los procesos, organización y relaciones de Tl & 5 & Alto \\
\hline PO5 Administrar la inversión en Tl & 5 & Alto \\
\hline PO6 Comunicar las aspiraciones y la dirección de la gerencia & 4 & Medio \\
\hline PO7 Administrar recursos humanos de $\mathrm{Tl}$ & 2 & Medio \\
\hline PO8 Administrar la calidad & 1 & Inmaterial \\
\hline PO9 Evaluar $\mathrm{y}$ administrar los riesgos de $\mathrm{Tl}$ & 5 & Alto \\
\hline PO10 Administrar proyectos & 3 & Medio \\
\hline \multicolumn{3}{|c|}{ ADQUIRIR E IMPLEMENTAR } \\
\hline Al1 Identificar soluciones automatizadas & 1 & Inmaterial \\
\hline Al2 Adquirir y mantener software aplicativo & 5 & Alto \\
\hline Al3 Adquirir y mantener infraestructura tecnológica & 5 & Alto \\
\hline Al4 Facilitar la operación y el uso & 5 & Medio \\
\hline Al5 Adquirir recursos de $\mathrm{Tl}$ & 1 & Inmaterial \\
\hline Al6 Administrar cambios & 3 & Medio \\
\hline Al7 Instalar y acreditar soluciones y cambios & 2 & Bajo \\
\hline \multicolumn{3}{|c|}{ ENTREGAR Y DAR SOPORTE } \\
\hline DS1 Definir y administrar los niveles de servicio & 2 & Medio \\
\hline DS2 Administrar los servicios de terceros & 4 & Alto \\
\hline DS3 Administrar el desempeño y la calidad & 2 & Medio \\
\hline DS4 Garantizar la continuidad del servicio & 5 & Alto \\
\hline DS5 Garantizar la seguridad de los sistemas & 5 & Alto \\
\hline DS6 Identificar $y$ asignar costos & 2 & Medio \\
\hline DS7 Educar y entrenar a los usuarios & 5 & Alto \\
\hline DS8 Administrar la mesa de servicio y los incidentes & 1 & Inmaterial \\
\hline DS9 Administrar la configuración & 3 & Medio \\
\hline DS10 Administrar los problemas & 4 & Alto \\
\hline DS11 Administrar los datos & 3 & Medio \\
\hline DS12 Administrar el ambiente físico & 2 & Bajo \\
\hline DS13 Administrar las operaciones & 1 & Inmaterial \\
\hline \multicolumn{3}{|c|}{ MONITOREAR Y EVALUAR } \\
\hline ME1 Monitorear y evaluar el desempeño de TI & 5 & Alto \\
\hline ME2 Monitorear $y$ evaluar el control interno & 4 & Medio \\
\hline ME3 Garantizar el cumplimiento regulatorio & 1 & Inmaterial \\
\hline ME4 Proporcionar gobierno de Tl & 3 & Bajo \\
\hline
\end{tabular}

\section{C. ISO 20000 Y 27000: GESTIÓN DE LA CALIDAD DE LOS SERVICIOS DE TI}

Los estándares internacionales ISO 20000 e ISO 27000 permiten garantizar la prestación satisfactoria de los servicios de TI y la gestión que se realice sobre estos, para lograr la satisfacción de los usuarios.

8. ISO 20000

IMPLEMENTACIÓN DE PROCEDIMIENTOS DE GOBERNABILIDAD TI EN LA RED DE INVESTIGACIÓN DE TECNOLOGÍA AVANZADA BASADO EN ITIL, COBIT Y LA ISO 20000-27000 Roberto Ferro Escobar / Giovanny Mauricio Tarazona Bermúdez / Gabriel Andres Alzate Acuña 
Para la implementación de esta norma en la Universidad Distrital se deben seguir un procedimiento con base a:

- $\quad$ Se debe conformar un equipo conocedor del modelo organizacional y tener experiencia en las áreas relacionadas con las TIC, para garantizar una correcta implementación, seguimiento, control y mejora.

- Definir y suministrar un catálogo de servicios de TI centrado en las necesidades de la comunidad universitaria con base en Acuerdos de Niveles de Servicio.

- $\quad$ Realizar un Sistema de Gestión de Seguridad de la Información acorde con el Sistema de Gestión de Información Institucional.

- $\quad$ La Universidad debe reforzar unas políticas, procesos, procedimientos y guías que disminuyan la incertidumbre en cuanto a las actividades e inversiones en materia de los servicios de TI.

ISO 27000

La Universidad deberá tener en cuenta la norma ISO 27000 para la Seguridad de la Información y podrá implementarse siguiendo las siguientes recomendaciones:

- Gestión de Activos: Cada dependencia, bajo supervisión del Comité de Seguridad de la Información, debe elaborar y mantener un inventario de los activos de información que posean.

- Gestión de Incidentes: El personal de la Universidad debe reportar con diligencia, prontitud y responsabilidad presuntas violaciones de seguridad a través de su jefe de dependencia a la Oficina Asesora de Sistemas o la Red de Datos UDNET. En casos especiales dichos reportes podrán realizarse directamente a la Oficina Asesora de Sistemas, la cual debe garantizar las herramientas informáticas para que formalmente se realicen tales denuncias.

- Recursos Humanos: Todo el personal de la Universidad Distrital Francisco José de Caldas, cualquiera sea su situación contractual, la dependencia a la cual se encuentre adscrito y el nivel de las tareas que desempeñe debe tener asociado un perfil de uso de los recursos de información, incluyendo el hardware y software asociado. La Oficina Asesora de Sistemas en coordinación con la Red de Datos UDNET deben mantener un directorio completo y actualizado de tales perfiles.

- $\quad$ Seguridad Física: La Red de Datos UDNET debe asegurar que la infraestructura de servicios de TI este cubierta por mantenimiento y soporte adecuados de hardware y software.

\section{CONCLUSIONES}

Pues bien, después de verificar los procesos de estándares para gobernabilidad basados en ITIL, ISO 20000, ISO 27000 y COBIT, es viable que la implementación de los mismos ayude a mejorar y apoyar la gestión de los recursos TI, la gestión de los servicios TI y la seguridad de la información en todos los procesos de la Red de Investigación de Tecnología avanzada RITAUD. Es esta forma se logra la alineación con el Plan Estratégico de Tecnología Informática y con el Plan de Desarrollo de la Universidad, generando mejores tiempo de respuesta a la comunidad académica e investigativa, reducción de costos, mitigación de riesgos, mayor calidad en los servicios.

\section{Referencias}

[1] Universidad Distrital Francisco José De Caldas. "Plan Estratégico de Desarrollo 2007- 2016". Grupo Editorial Gaia. Bogotá. 2006. p. 97

[2] Paulson, Linda Dailey., "Internet2 Upgrades Backbone", available online ieeexplore.ieee.org/ie/2/20936/00970552. pfd"

[3] Red Universitaria Metropolitana de Bogotá, RUMBO, http://www.rumbo. edu.co/.

[4] Red Nacional de Tecnología Avanzada, http://www.renata.edu.co/

[5] CLARA Red Latinoamericana de Redes Avanzadas, http://www.redclara.net/

[6] Red de Investigaciones de Tecnologías Avanzadas, http://sig-terminal.udistrital. edu.co/

[7] Isaca, Cobit 5, Un Marco de Negocio para el Gobierno y la Gestión de las TI de la Empresa, 2012, http://www. 
isaca.org/COBIT/Documents/COBIT5Framework-Spanish.pdf

[8] Nuevas tecnologías y Negocios, Baquia, http://www.baquia.com/blogs/seguridad/ posts/2013-02-28-la-hora-de-elevar-elspeech-de-ti

[9] A. Cartlidge, IT INFRASTRUCTURE LIBRARY, An Introductory Overview of ITIL ${ }^{\circledR}$ V3,2007. https://www.bestmanagement-practice.com/gempdf/ itSMF_An_Introductory_Overview_of ITIL_V̄3.p $\overline{d f}$

[10] F. Ferrer, Consideraciones prácticas para una implementación exitosa de COBIT, FERROL, International group, 2011, http://www.proactivanet.com/UserFiles/ File/FERROLIG-TCM-COBIT.pdf

[11] Normas ISO, 20000, http://www.normasiso.com/etiqueta/iso-20000

[12] Normas ISO, 27000, El estándar de seguridad de la información, http://www. iso27000.es/download/doc iso27000 all. pdf

[13] Universidad Distrital Francisco José de Caldas. Política de Seguridad de la Información y su implementación, Comité de Informática, ttps://portalws. udistrital.edu.co/CIT/documentos/ NORMATIVIDAD/politica_seguridad/ archivos/Politica_para_Seguridad_ Informacion_Version_0.0.1.0.pdf

[14] TCM Tecnologías con Clase Mundial, implantación de nuevos procesos y tecnologías, Bogotá Colombia, http:// www.tcm-ti.com/itil.html

[15] G. Quintero, Implementación de procedimientos ITIL v3.0 en la gestión de TI de la Universidad del Valle, 20082011. ACTAS TICAL 2011. De http:// tical_2011.redclara.net/doc/ACTAS TICAL2011.pdf

[16] COMITÉ DE INFORMÁTICA. (2012). Plan Maestro de Informática y Telecomunicaciones. https://portalws. udistrital.edu.co/CIT/documentos/ DOCUMENTOS TRABAJO/PMITUD/archivos/PMITUD 2012-2018 Aprobado.pdf 